A. P. Chetverikov, W. Ebeling, E. Schöll, M. G. Velarde

\title{
Excitation of solitons in hexagonal lattices and ways of controlling electron transport
}

Journal article | Accepted manuscript (Postprint)

This version is available at https://doi.org/10.14279/depositonce-9756

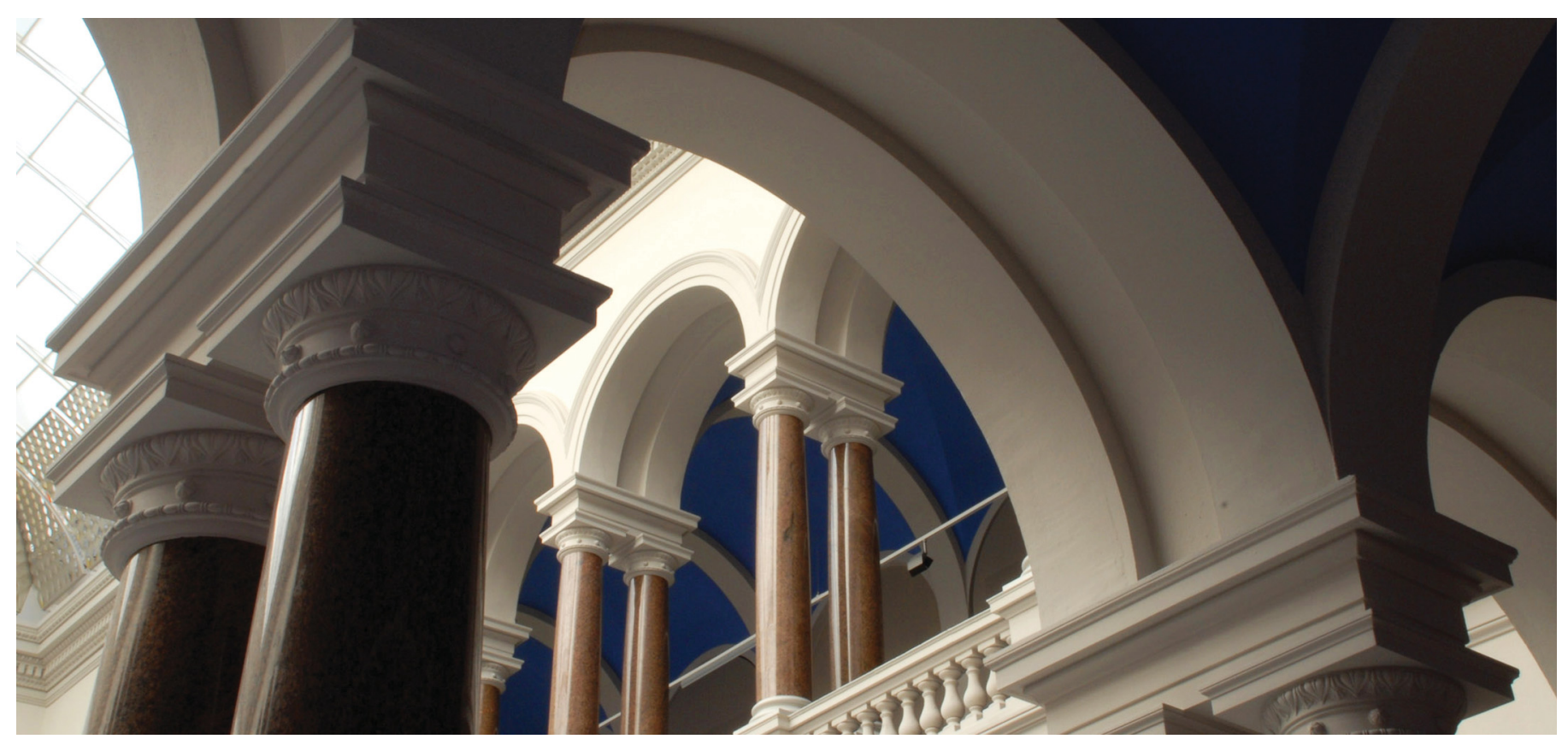

This is a post-peer-review, pre-copyedit version of an article published in International Journal of Dynamics and Control. The final authenticated version is available online at:

http://dx.doi.org/10.1007/s40435-017-0383-x.

Chetverikov, A.P., Ebeling, W., Schöll, E. et al. Excitation of solitons in hexagonal lattices and ways of controlling electron transport. Int. J. Dynam. Control 6, 1376-1383 (2018).

https://doi.org/10.1007/s40435-017-0383-x 


\title{
Excitation of solitons in hexagonal lattices and ways of controlling electron transport
}

\author{
A. P. Chetverikov \\ Faculty of Physics, Saratov National Research State University, \\ Astrakhanskaya 83, 410012 Saratov, Russia \\ W. Ebeling \\ Institute of Physics, Humboldt-University Berlin, \\ Newtonstraße 15, D-12489 Berlin, Germany \\ E. Schöll \\ Institut für Theoretische Physik, Technische Universität Berlin, \\ Hardenbergestraße 36, D-10623 Berlin, Germany \\ M. G. Velarde \\ Instituto Pluridisciplinar, Universidad Complutense, \\ Paseo Juan XXIII, 1, 28040 Madrid, Spain \\ (Dated: 01.10.2016)
}

\begin{abstract}
We construct metastable long-living hexagonal lattices with appropriately modified Morse interactions and show that highly-energetic solitons may be excited moving along crystallographic axes. Studying the propagation, their dynamic changes and the relaxation processes it appears that lump solitons create in the lattice running local compressions. Based on the tight-binding model we investigate the possibility that electrons are trapped and guided by the electric polarization field of the compression field of one soliton or two solitons with crossing pathways. We show that electrons may jump from a bound state with the first soliton to a bound state with a second soliton and changing accordingly the direction of their path. We discuss the possibility to control by this method the path of an excess electron from a source at a boundary to a selected drain at another chosen boundary by following straight pathways on crystallographic axes.
\end{abstract}

PACS numbers: 05.45.YvSolitons, 63.20.RyAnharmonic lattice models, 71.38.-kPolarons and electronphonon interactions, 73.63.-bElectric transport in nanoscale materials

\section{Introduction}

The present work is motivated by two timely events. On the one hand nowadays the world of atomically thin two-dimensional (layered) materials and some metamaterials is becoming of extraordinary interest [1-11]. On the other hand, a useful tool for the exploration of two-dimensional layered materials are (linear and nonlinear) surface acoustic waves (SAW) [12-21]. Linear and nonlinear SAW propagating in a homogeneous elastic medium, piezoelectric, or otherwise, exhibit no dispersion. If the medium is nonlinear, as e.g. in anharmonic crystal lattices, an initial sinusoidal SAW can create higher harmonics which may grow without being inhibited by dispersion. Dispersion can be introduced by coating the medium with a thin film of another material with elastic and structural/mechanical properties different from those of the substrate. Then by an appropriate choice of the film thickness, the effects of nonlinearity and dispersion can locally balance each other thus sustaining solitons as long ago Nayanov and others observed [13-21]. The SAW offer the possibility of acoustic charge transport in semiconductor heterostructures and even the possibility of charge transfer on demand. Such form of transport is reminiscent of electron "surfing" [22-26]. As high amplitude SAW tend to deform to sawtooth shape and eventually break, the suggestion coming from our theory is that for electron surfing, irrespectively of the scale involved, solitons should be better carriers than linear waves. In particular we are interested in the possibility of controlling the path of electrons at the nanoscale.

In view of the above, as a paradigmatic model here we study a special representative of the class of hexagonal (2d) lattices (Fig. 1) by focusing on a very simple model of nonlinear excitations coupled to tight-bound electrons [27].

\section{Modeling a long living metastable hexagonal} lattice

We consider systems of $10^{2}-10^{3}$ atoms on a plane (up to 1600) interacting with a Morse potential where atoms repel each other exponentially and attract each other with weak dispersion forces (Fig. 2). Such potential is of current use in physics and chemistry and it was the first interatomic potential based on quantum mechanics. In addition the lattice units interact with one or a few added excess electrons. Now before proceeding to the hexagonal lattice it is worth recalling that for a triangular lattice, which is topologically the basis of $2 \mathrm{~d}$ lattices, the Morse potential that rules out overcounting the unphysical influence of neighbors outside the first 
neighborhood of each atom is $[27,28]$

$$
V(r)=D\{\exp [-2 b(r-\sigma)]-2 \exp [-b(r-\sigma)]\},
$$

where

$$
r=\left|r_{n}-r_{j}\right|
$$

Yet for a realistic description of some quite significant hexagonal crystals like a graphene lattice, the Morse interaction is not sufficient. For the latter Savin et al. use a composite expression consisting of at least 5 components [29]:

$$
U=\sum U_{1}+\sum U_{2}+\sum U_{3}+\sum U_{4}+\sum U_{5}
$$

The first part is a sum of Morse potentials eq. (1) with the parameters

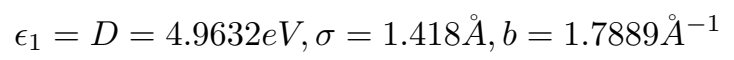

The contribution $U_{2}$ models the deformation energy of the angles between the valence bonds having the form

$$
\begin{array}{r}
U_{2}\left(\mathbf{r}_{1}, \mathbf{r}_{2}, \mathbf{r}_{3}\right)=\epsilon_{2}\left[\cos (\varphi)-\cos \left(\varphi_{0}\right)\right]^{2}, \\
\left.\left.\cos (\varphi)=\left(\mathbf{r}_{3}-\mathbf{r}_{2}, \mathbf{r}_{1}-\mathbf{r}_{2}\right) /\left(\mid \mathbf{r}_{3}-\mathbf{r}_{2}\right)|\cdot| \mathbf{r}_{2}-\mathbf{r}_{1}\right) \mid\right)
\end{array}
$$

with the energy $\epsilon_{2}=1.3143 \mathrm{eV}$. For our purpose here we will use only the first part in a modified version and show that there exists already a long living metastable hexagonal lattice. There is no need of making explicit the other components which are responsible for the stability of the stable real ground state of the hexagonal lattice. Our basic assumption is that for the investigation of very short excitations as studied here, a metastable hexagonal lattice suffices.

3. Excitation of solitons in the hexagonal lattice

The excitations of the lattice are created by external perturbations like mechanical or electrical shocks generated, e. g., by contacts of the tip of an electron field microscope with a suitable anharmonic crystal lattice. In our molecular dynamics computer simulations we shall use a code which instead of the coordinates $x_{j}, y_{j}$ uses the complex coordinates

$$
Z_{j}=x_{j}+i y_{j}
$$

which has the advantage that distances may be easily expressed by absolute values of $Z$, e.g.,

$$
\left|\mathbf{r}_{1}-\mathbf{r}_{2}\right|=\left|Z_{1}-Z_{2}\right|
$$

and the angles by imaginary parts of logs, e.g.,

$$
\varphi\left(\mathbf{r}_{1}, \mathbf{r}_{2}, \mathbf{r}_{3}\right)=\operatorname{Im}\left(\ln \frac{\left|Z_{3}-Z_{2}\right|}{\left|Z_{1}-Z_{2}\right|}\right) .
$$

The complex configuration $Z$ (with $x, y$ measured in units of $\sigma$ ) simplifies all calculations. In order to focus on the generic features we will neglect later all contributions beyond $U_{1}$. This is a serious limitation of our approach which needs some comments. Indeed, the ground state of Morse-type potentials is always a triangular lattice. Therefore as already noted hexagonal configurations with a potential of type $U_{1}$ represent a local minima of the potential landscape. In order to make the configuration stable long enough to allow for transport we need at least the angle-dependent part $U_{2}$. However for a first study of excitations with characteristic scale much shorter than the relaxation to the real (triangular) ground state, a model based solely on $U_{1}$ might suffice. For such a purpose we play with the stiffness and the cut-off radius of the potential. According to the Savin model, the stiffness of graphene is around $b \sigma \simeq 2.5$. In order to create sufficiently deep side-minima in the hexagonal configuration and allow for solitonic excitation, we fix here the effective stiffness parameter in the simplified model to a higher value $b \sigma=8$. To describe a hexagonal lattice, instead of (1) we use for the hexagonal lattice

$$
\begin{array}{r}
V(r)=D\{\exp [-2 b(r-\sigma)]-2 \exp [-b(r-\sigma)]\} \\
\cdot\{1+\exp [(r-d) / 2 \nu]\}^{-1}
\end{array}
$$

This guarantees that, unlike Eq. (1), the force of interaction and the derivative of this force do not have a strong discontinuity at the cut-off point. The cut-off "interaction radius" is assumed to be $1.5 \sigma$, together with parameter values $d=1.35 \sigma$ and $\nu=0.025 \sigma$. In Fig. 2 the original Morse potential (1) is shown in green (lower dotted line) and the modified one (10) in red (upper solid line). Beyond the cut-off radius the potential is set to zero. With this choice of the potential we expect to create side-minima of the potential landscape (see Fig. 1) which are stable long enough even under dynamic conditions and allow us to study the lattice interaction with electrons. The Hamiltonian of our 2d lattice consists

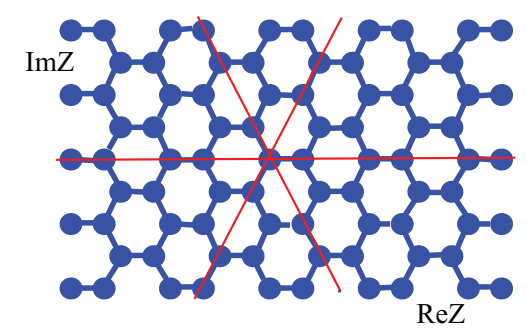

Figure 1: Scheme of a hexagonal lattice, represented in complex lattice coordinates $Z$. The crystallographic axes along which (lump) solitons may propagate are shown as thin (red) lines.

of a classical lattice component $H_{a}$, and the contribution of the electrons $H_{e}$, which includes the interactions with the lattice deformations. For the lattice part, the 


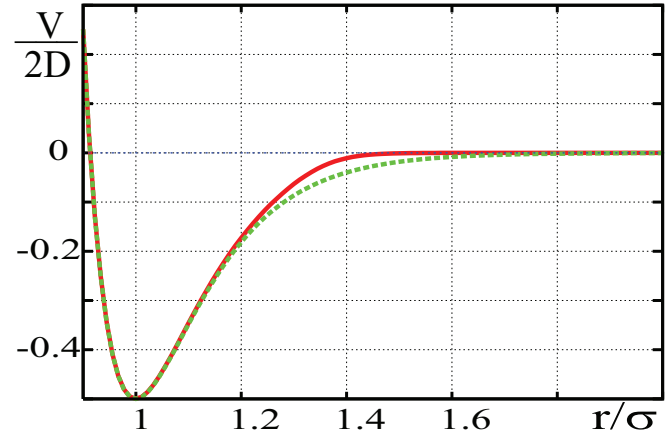

Figure 2: Interaction between lattice particles: Comparison of the original Morse potential (green, lower dotted line) with the "truncated" Morse potential (red upper line) used for our computer simulations (parameter values $b \sigma=8, d=1.35 \sigma$, $\nu=0.025 \sigma)$.

Hamiltonian is

$$
H_{a}=\frac{m}{2} \sum_{n} v_{n}^{2}+\frac{1}{2} \sum_{n, j} V\left(r_{n}, r_{j}\right) .
$$

The subscripts locate the atoms all with equal mass, $m$, at lattice sites and the summations run from 1 to $N$ $[27,28,30]$. For visualization and tracking the atomic electron densities we model the atoms as little spheres with "core" electrons represented by a Gaussian distribution centered at each lattice site (Fig. 3):

$$
\rho(Z, t)=\sum_{\left|Z-Z_{n}(t)\right|<1.5} \exp \left[-\frac{\left|Z-Z_{n}(t)\right|^{2}}{2 \lambda^{2}}\right] .
$$

In order to follow the evolution of localized excitations

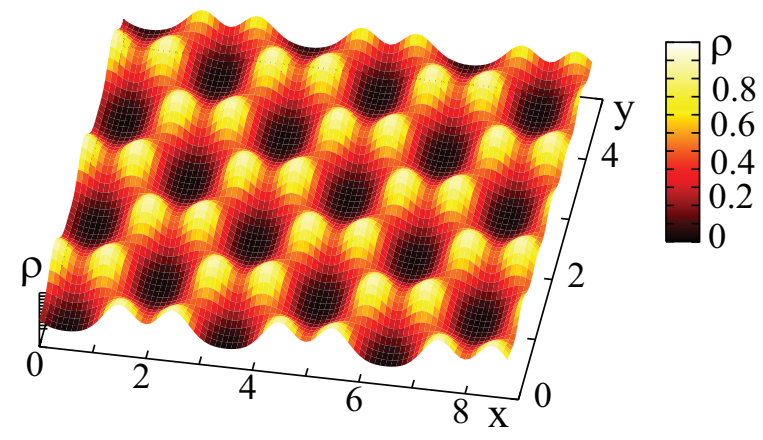

Figure 3: The Gaussian core electron probability $\rho$ visualizing the hexagonal lattice structure at rest.

from now on we only plot the difference $\rho_{e x}$ between the density of the excited lattice and the equilibrium lattice which is taken as reference zero value. To study the nonlinear excitations of the lattice and the possible electron transport we need the coordinates of the (point) particles at each time and the interaction of lattice deformations with electrons. Coordinates of particles are obtained by solving the equations of motion of each particle under the influence of all possible forces. The latter may also include weak friction and random forces as accounted by a Langevin model, not to be considered here. Using complex coordinates $Z=x+i y$ the Newtonian deterministic equations corresponding to the lattice Hamiltonian (11) are

$$
\frac{d^{2} Z_{n}}{d t^{2}}=\sum_{k} F_{n k}\left(\left|Z_{n k}\right|\right) z_{n k}
$$

where index $n$ identifies a particle among all $N$ particles of the ensemble. Further $Z_{n k}=Z_{n}-Z_{k}$ and $z_{n k}=\left(Z_{n}-Z_{k}\right) /\left|Z_{n}-Z_{k}\right|$ is the unit vector defining the direction of the interaction force $F_{n k}$, corresponding to the Morse potential, between the $n$-th and the $k$-th atoms in the lattice. As mentioned above, we consider dimensionless spatial coordinates rescaled with $\sigma$ as unit length. Time is normalized to the inverse frequency of linear oscillations near the minimum of the Morse potential well, $\omega_{0}^{-1}$, whereas energy is scaled with $2 D$. In view of the above only those lattice units with coordinates $Z_{k}$, satisfying the condition $\left|Z_{n}-Z_{k}\right|<1.5$, are taken into account in the sum in Eq. (13). In computer simulations the interaction of lattice units is considered to take place inside a rectangular cell $L_{x} \cdot L_{y}$ with periodic boundary conditions and depending on the symmetry of an initial distribution of units and their number $N$. The initial condition is defined by the regular hexagonal configuration. Using data about trajectories of particles $Z_{n}(t)$ and their velocities we calculate the lattice atom distribution described by the core electron probability density $\rho(Z, t)$.

In Fig. 4 we show a track of the running excitation (in a "bubble chamber representation") which was created by pushing just one atom in the direction of the crystallographic axis $x$. We show the space and time evolution of the initial soliton density peak for the time interval $\Delta t=30$.

The soliton which is moving along a crystallographic axis was excited by a strong pulse of velocity $v_{0}$ imposed at $t=0$ to the 2 nd atom in the 10 th row with rather high energy (relative to 2D) $m v_{0}^{2} / 2$. The highly energetic soliton excited in this way is quite long lasting in its motion along the chosen crystallographic axis. In some sense such a soliton is like a crowdion or a mobile breather (see, e.g., [31-33]). Transverse excitations and thermal collisions do not play a significant role for $\Delta t<20$, then the transverse distortion starts to grow. The snapshot of the lattice state shows that the head of the soliton is already at position 30 but the changes in the lattice are still seen along the path. The very left dark points remember the initial kick. What follows is the dynamic reconstruction of the lattice including also emission of phonons [33]. Only after a finite time the lattice returns more or less to the ordered crystalline state. In the intermediate time we can say that the lattice is in a non-crystalline state and unable to allow for solitons to 

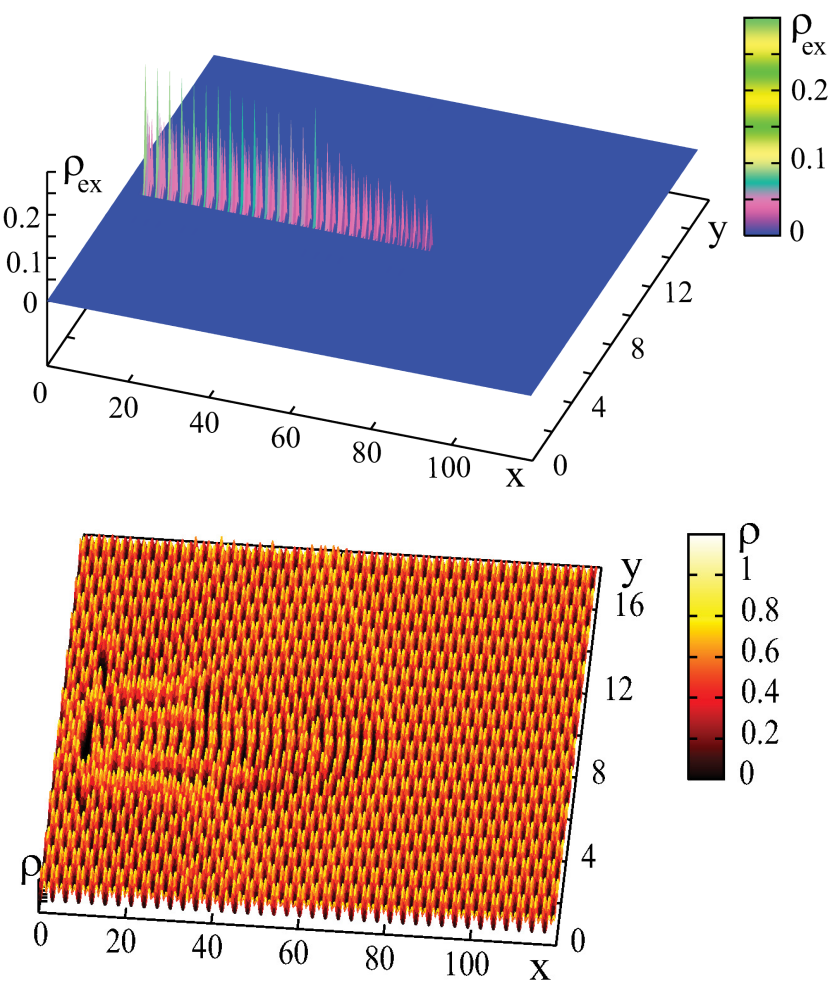

Figure 4: Soliton excitation in a hexagonal Morse lattice. $\mathrm{Cu}-$ mulative series of snapshots of the lattice atoms in the course of time. A track of the excitation is seen in "bubble chamber representation" of the running soliton density $\rho_{e x}$ for a time interval $\Delta t=30$ in a cumulative sequence as time proceeds. Upper panel: The soliton is excited by a strong pulse with velocity $v_{0}=2$ (in units $\omega \sigma$ ) imposed to one atom in a crystallographic direction. Lower panel: The lattice state of deformation at time $t=30$. We note that for $t>20$ the lattice deformation is already very much extended laterally. Lattice size: $N=1600$.

cross the path. Any second soliton which crosses the trace of a soliton in the delay time will get stuck. Let us show next that this effect makes it possible to control the path of an electron. We show the dynamics changes in the lattice around a passing soliton or solectron lead locally to some reversible local perturbation creating a special non-crystalline transient state which is essentially connected with phonon radiation (Fig. 4). After a finite time which is larger than 40 time units in our examples the lattice returns to an ordered lattice state. In the intermediate time the lattice is not able to support solitons which are crossing the path. This time delay can be used to advantage for a form of control of electron transport.

From the length of the cumulative path and the time interval we may estimate the velocity. It appears that this strong local compression moves with velocity exceeding the sound velocity with a lifetime of at least several time units. Indeed, they advance for a few picoseconds with nearly unaltered profile and precisely this robustness is the reason why we can identify them by the proposed visualization method. Note that the $2 \mathrm{~d}$ solitons observed here are similar to the so-called lump solutions of the Kadomtsev - Petviashvili equation $[30,34,35]$.

\section{Electron hopping dynamics in the hexagonal lattice}

Let us now focus on the role played by one or several non-interacting electrons embedded into the atomic lattice, possibly as a result of doping, injection or photoexcitation. Following Davydov [36], a soliton is connected with a deformation density of the lattice along its path. In order to study the evolution of the quantum states of the additional electrons interacting with the atoms in the 2d-lattice, we use the tight binding approximation (TBA) [27]

$$
H_{\mathrm{el}}=\sum_{n} E_{n} c_{n}^{+} c_{n}+\sum_{n, n^{\prime}} t_{n, n^{\prime}}\left(\mathbf{r}_{n^{\prime}}-\mathbf{r}_{n}\right) c_{n^{\prime}}^{+} c_{n}
$$

where $t_{n, n^{\prime}}$ is the transition matrix. Here we assume only one quantum state of electrons per site and transitions between $\mathbf{r}_{n}$ and $\mathbf{r}_{n}^{\prime}$. The transition matrix elements $t_{n, n^{\prime}}$ depend on the atomic distances, $t_{n, n^{\prime}}=t\left(\mathbf{r}_{n^{\prime}}-\mathbf{r}_{n}\right)$. We assume an exponential expression for the transition probabilities [27]

$$
t_{n, n^{\prime}}=V_{0} \exp \left[-\alpha_{h}\left|\mathbf{r}_{n}-\mathbf{r}_{n^{\prime}}\right|\right]
$$

The range parameter $\alpha_{h}$ can be related to the tunneling probability that decreases exponentially with distance. A full quantum mechanical description of the electrons in the field of the fast changing lattice is rather difficult. To simplify this situation we postulate as model for the electron dynamics $N$ Schrödinger equations in TBA for the complex amplitudes $c_{n}$ given in dimensionless units by $[37-39]$

$$
i \frac{d c_{n}}{d t}=\epsilon_{0} c_{n}-\tau \exp (\alpha b \sigma) \sum_{\left|Z_{n}-Z_{m}\right|<1.5} c_{m} \exp \left(-\alpha\left|Z_{n}^{\prime}-Z_{m}^{\prime}\right| 016\right)
$$

where the amplitude belongs to the quantum state of atom $n(n=1 \ldots N)$ located at $Z_{n}=x_{n}+i y_{n}$, further we use $\tau=V_{0} / \hbar \omega_{0}, \alpha=\alpha_{h} b^{-1}, Z^{\prime}=b \sigma Z$.

We do not assume any regular order of the atoms, except at $t=0$, where an hexagonal lattice configuration is assumed. The electrons may hop between the lattice sites, the constant energy $\epsilon_{0}$ of electrons at lattice site $n$ is irrelevant for the dynamics. The sum $n \neq m$ in the transition term is extended over pairs with distance smaller than $1.5 \sigma$. Quantum transitions occur preferentially between nearby lattice sites. Then the complex Newtonian 

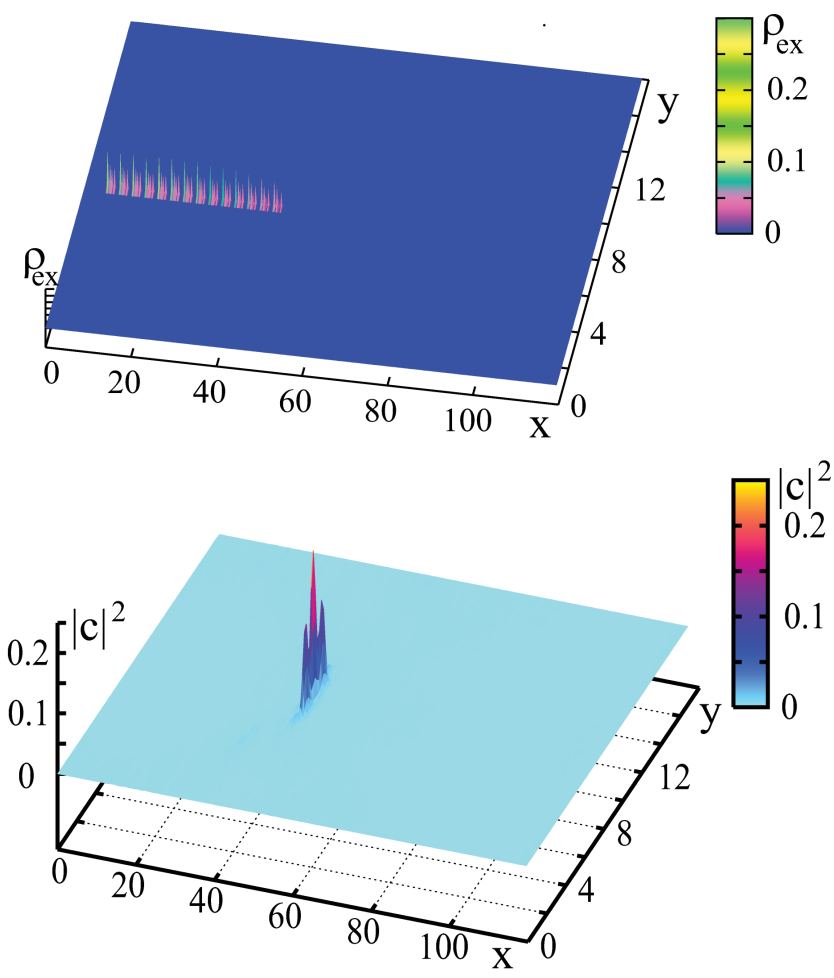

Figure 5: Hexagonal Morse lattice with a soliton generated at $t=0$ by a strong kick $v_{0}=2$ at left boundary. At the same time a Gaussian electron is generated and placed near to the soliton. Upper panel: The lattice state is presented as a cumulative snapshot at time $t=15$. Lower panel shows the external electron probability density at $t=15$. $N=$ $1600, b \sigma=8, v_{0}=2$.

equations read:

$$
\begin{aligned}
& \frac{d^{2} Z_{n}^{\prime}}{d t^{2}}=\sum_{\left|Z_{n}-Z_{m}\right|<1.5}\left[\exp \left(b \sigma-\left|Z_{n}^{\prime}-Z_{m}^{\prime}\right|\right)\right. \\
& \cdot\left(1-\exp \left(b \sigma-\left|Z_{n}^{\prime}-Z_{m}^{\prime}\right|\right)+2 \alpha V \exp \left(\alpha\left(b \sigma-\left|Z_{n}^{\prime}-Z_{m}^{\prime}\right|\right)\right)\right. \\
& \cdot \operatorname{Re}\left(c_{n} c_{m} *\right) \frac{Z_{n}-Z_{m}}{\left|Z_{n}-Z_{m}\right|} .
\end{aligned}
$$

$\left(V=V_{0} /(2 D)\right)$. We assume that the mechanical excitations are strong enough to dominate the evolution of the system. Throughout the simulations presented below we fix the hopping parameters to

$$
\tau=10, \alpha=1, V=1 \text {. }
$$

Here we work in the region of low enough temperatures, where the temperature influence is negligible, and use as model for the electron dynamics the $N$ Schrödinger equations (16). Note that the approach may be extended to the motion of electron pairs as shown, e.g., in [40]. For higher temperatures one useful approach is to consider the electron dynamics as a Monte Carlo process.

\section{Controlling the path of an electron as soliton assisted motion}

Our idea of controlling the path of electrons is based on the properties of the deformations explained in Section 3. Just in the moment before the soliton/solectron crosses a certain point on its path on a crystallographic axis (which is here parallel to the lower boundary) we let another soliton (with otherwise same parameter values) starting from the lower boundary cross its path. The first soliton will stop for a while since it moves to a messy (noncrystalline) lattice region. However, the electron sitting on the first soliton, due to its delocalized nature, may "see" the potential of the second soliton which just came along and jumps onto it. This is illustrated in Figs. 6, 7 and 8 .

In the electron trapping process, the local lattice compressions significantly deform the potential landscape acting on added excess electrons and create a moving guiding well or trap (this is indeed the dynamic solectron bound state). There is also a feedback of the concentration of electron density on the lattice deformation (polaron effect) which in view of earlier discussion we neglect in a first approximation. Indeed, in the supersonic case, for the given parameter values, this feedback is rather small, changing the results by less than a few percent [41]. Generally, the electrons tend to be trapped in the regions of maximal density of lattice atoms created by the local compressions and then forced to move dynamically in a state bound to the soliton-like compressions which in $2 \mathrm{~d}$ is favored along the crystallographic axes.

Fig. 5 shows a soliton moving from left to right (carrying an electron). Without meeting an obstacle the electron will be carried along the crystallographic axis to the right boundary. However, if the soliton on its path, as shown in Figs. 6-8, encounters another soliton at an angle of $60^{\circ}$ (or $120^{\circ}$, respectively) a reorientation starts. As the former soliton cannot proceed properly due to the messy locally non-crystalline state in the lattice after passing ) the second soliton crossing from below (which is similar to a local "melting") then this second soliton dominates the dynamics. Then the electron tends to abandon the bound state with the former soliton (its first partner) and is prone to be bound to the second, now dominant soliton, hence forming a new solectron. Consequently, the crossing of two solitons permits to bring an electron from the given source to a chosen drain either on its unperturbed first path or near the right upper corner or the left upper corner of the lattice, respectively.

\section{Conclusion}

We have proposed the effect of controlling electron transport without external electric field in a (thin) two-dimensional hexagonal molecular lattice. The source-drain channel for the electron is naturally conducted along one of three crystallographic axes by simply playing with two lattice solitons excited at 

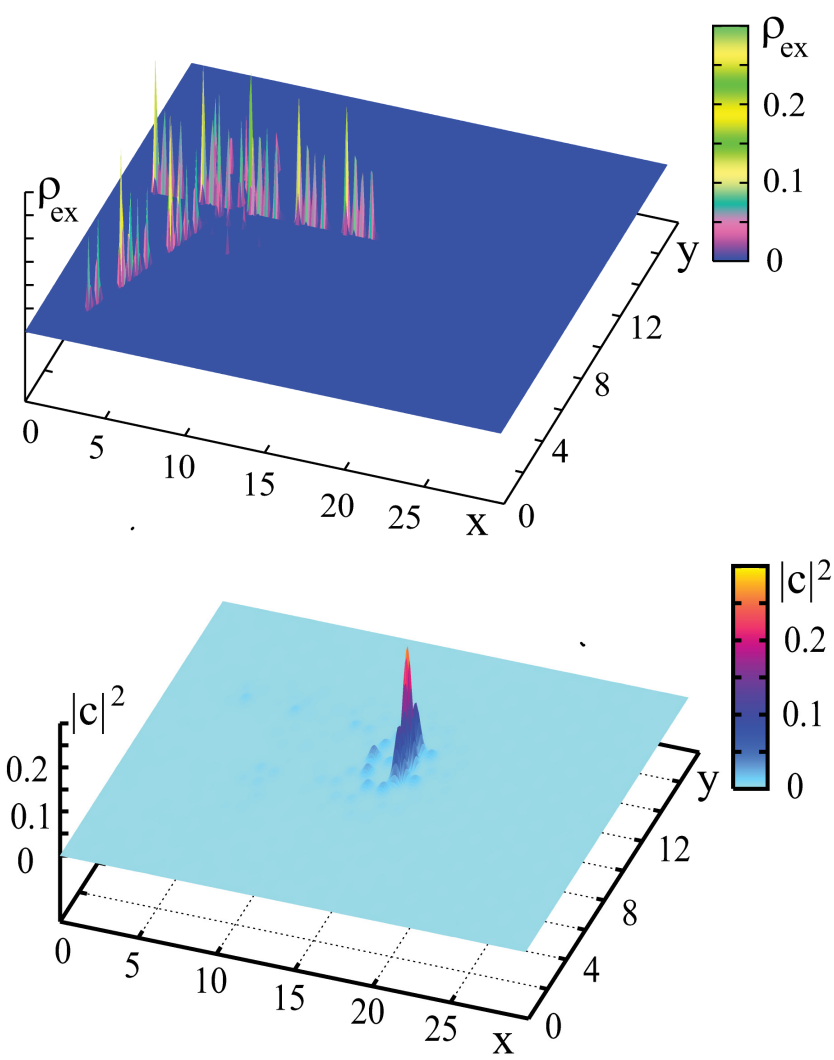

Figure 6: Crossing excitations in a hexagonal lattice. Soliton and electron initially placed at the left boundary (electron is placed nearby). The second soliton starts at the same time at $x=1$. We see that after crossing the path of the first soliton and the electron (hence already forming a solectron) the second soliton dies because it meets a strongly disturbed lattice and is unable to propagate further. The experiment corresponds to crossing with a soliton under angle of $60^{\circ}$. The deformation probability of the lattice $\rho_{e x}$ (in cumulative view) and the state density of the external electron $\left|c^{2}\right|$ are shown at $t=5$. Accordingly, the initial solectron continues its path without showing any visible alteration. The probability density of the external electron arrives finally at a place (a chosen drain) at the right boundary. Lattice size: $N=400$.

appropriate positions with appropriate time delay. The whole procedure is essentially based on fine tuning of the relative times and positions of injection of solitons and electrons on the nanoscale.

The effect discussed here offers a novel way of controlling and transporting electrons from a given source to a chosen drain with no need of an imposed external electric field. The present computer experiments pertain to a nano-scale of a few hundred atoms in a plane and atomic time scales, but on a qualitative level they may also have promising applications to real experiments on a larger scale with nonlinear surface acoustic waves (SAW) in $2 \mathrm{~d}$ nanosystems and metameterials, e.g., graphene.
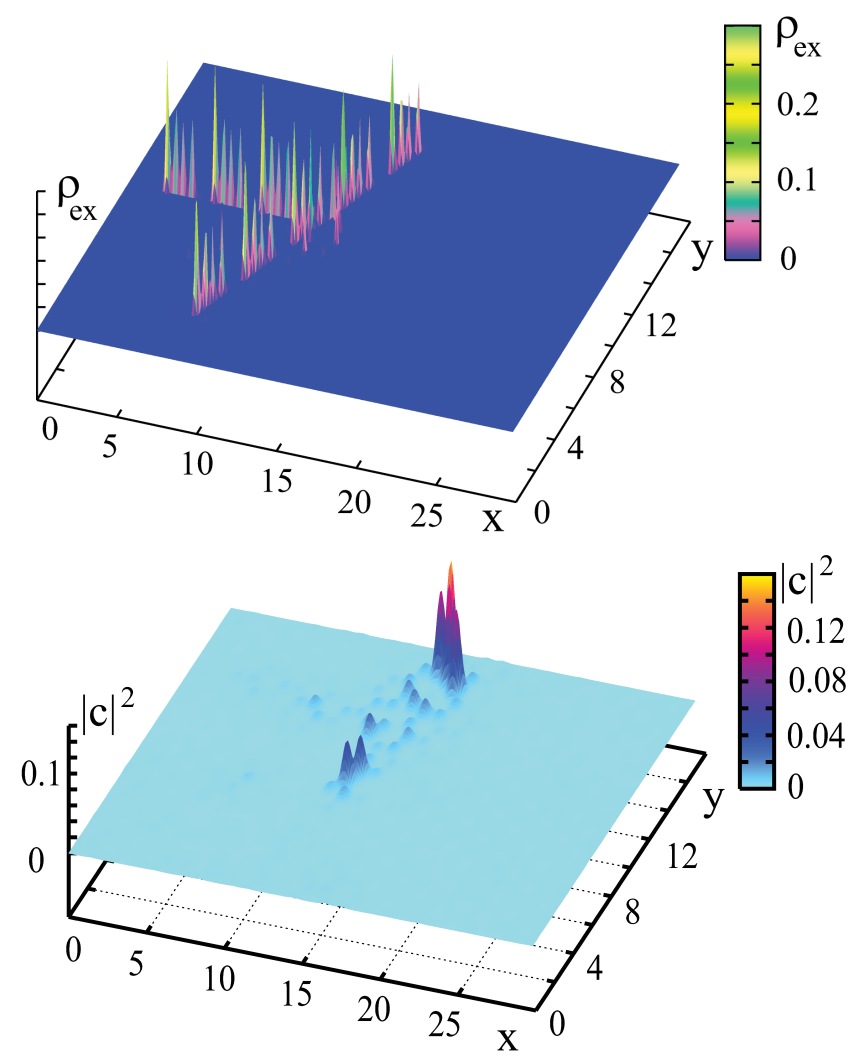

Figure 7: Same as Fig. 6, for the second soliton starting at the same time but now at $x=7.5$. We see that now after crossing the path of the first soliton and the electron the second soliton starts to move and even succeeds to take over the electron, thus creating a new solectron. On the other hand the first soliton finds now on its path a strongly disturbed lattice and is unable to propagate further. The experiment corresponds also to crossing with a soliton under angle of $60^{\circ}$ but later time. We see that the external electron changed its path and follows the second soliton to a place (a chosen drain) at the upper border. Lattice size: $N=400$.

Acknowledgement: The authors acknowledge fruitful discussions and correspondence with A. Knorr, R.P.G. McNeil, C. Ford, T. Meunier, and A. Wixforth. They also wish to thank V. I. Nayanov for his enlightening description of soliton SAW in nonlinearly elastic, piezoelectric $\mathrm{LiNbO}_{3}$ layers where wave dispersion able to balance nonlinearity of the substrate is monitored by depositing, via evaporation, $\mathrm{SiO}$ films of suitable thickness. This work was partially supported by the Collaborative Research Center 910: Control of self-organizing nonlinear systems: Theoretical methods and concepts of application (SFB 910) funded by Deutsche Forschungsgemeinschaft. A.P.C. acknowledges also support under project 16-12-10175 from the Russian Science Foundation. 

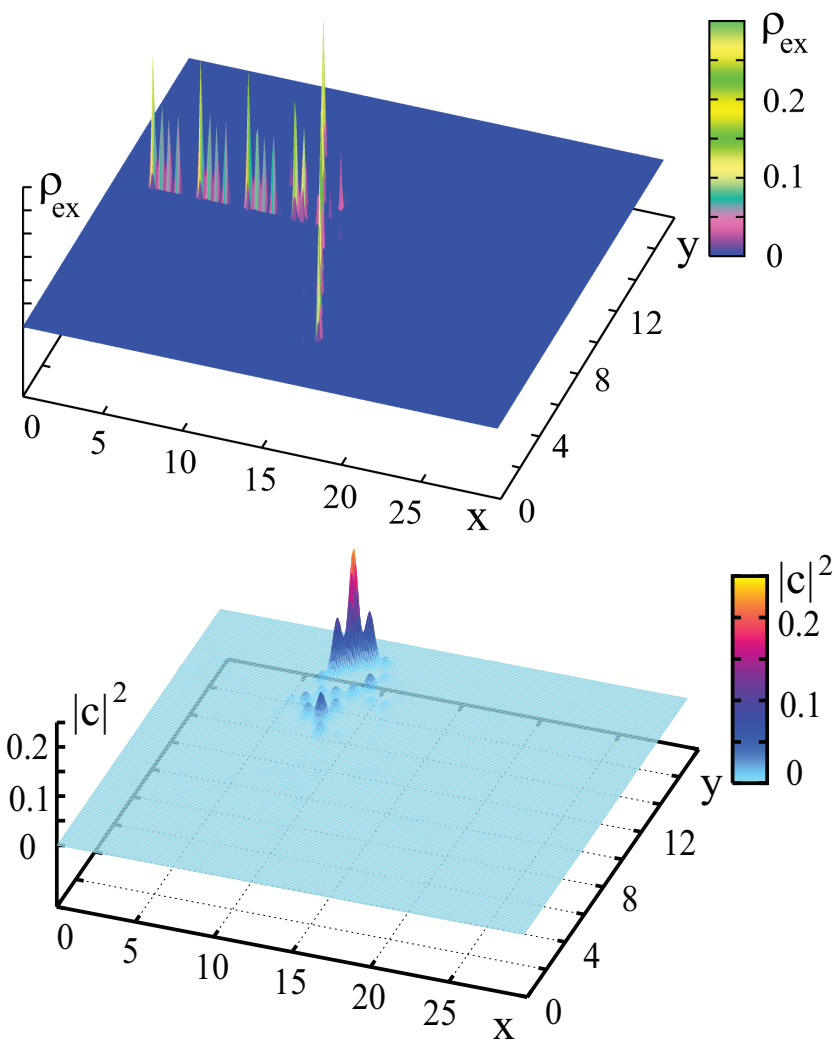

Figure 8: Same as Fig. 6, for the second soliton starting at the same time but now at $x=13.5$ on another crystallographic axis and crossing with the first soliton under angle of $120^{\circ}$, the crossing time being as before. We see again that now after crossing the path of the first soliton and the electron the second soliton continues to move and succeeds to take over the electron. On the other hand the first soliton finds again on its path a strongly disturbed lattice and is unable to propagate further. As before the external electron changed its path and follows the second soliton to a place (now a different drain) at the upper boundary. Lattice size: $N=400$.

[1] B. Radisavljevic, A. Radenovic, J. Brivio, V. Giacometti, A. Kis, Nat. Nanotechnol. 6, 147 (2011)

[2] A.C. Ferrari et al., Nanoscale. 7, 4598 (2015)

[3] R. Hoffmann, Angew. Chem. Int. Ed. 52, 93 (2013)

[4] L. Tao, E. Cinquanta, D. Chiappe, C. Grazianetti, M. Fanciulli, M. Dubey, A. Molle, D. Akinwande, Nat. Nanotechnol. 10, 227 (2015)

[5] F.-F. Zhu, W.-J. Chen, Y. Xu, C.-I. Gao, D.-D. Guan, C.-H. Liu, D. Qian, S.-C. Zhang, J.-F. Jia, Nat. Mater. 14, 1020 (2015)

[6] Z. Insepov, E. Emelin, O. Kononenko, D.V. Roshchupkin, K.B. Tryshtykbayev, K.A. Baigarin, Appl. Phys. Lett. 106, 023505 (2015)

[7] A.K. Geim, Random Walk to Graphene, Nobel Lecture, December 8, 2010

[8] X. Du, I. Skachko, A. Barker, E.Y. Andrei, Nature Nan- otechnology 3, 491 (2008)

[9] K.S. Novoselov, D. Jiang, F. Schedin, T.J. Booth, V.V. Khotkevich, S.V. Morozov, A.K. Geim, PNAS 102, 10451 (2005)

[10] N. Engheta, R.W. Ziolkowski, Metamaterials: Physics and Engineering Explorations (Wiley, N.Y., 2006)

[11] S. Rudykh, M.C. Boyce, Phys. Rev. Lett. 112, 034301 (2014)

[12] M.J. Hoskins, H. Morko, B.J. Hunsinger, Appl. Phys. Lett. 41, 332 (1982)

[13] V.I. Nayanov, JETP Phys. Lett. 44, 314 (1986)

[14] A. Wixforth, J.P. Kotthaus, G. Weimann, Phys. Rev. Lett. 56, 2104 (1986)

[15] W.J. Tanski, S.W. Merritt, R.N. Sacks, D.E. Cullen, E.J. Branciforte, R.D. Caroll, T.C. Eschrich, Appl. Phys. Lett. 52, 18 (1988) 
[16] A.P. Mayer, Phys. Rep. 256, 237 (1995)

[17] M. Streibl, A. Wixforth, J.P. Kotthaus, A.O. Govorov, C. Kadow, A.C. Gossard, Appl. Phys. Lett 75, 4139 (1999)

[18] M. Rotter, A.V. Kalameitsev, A.O. Govorov, W. Ruile, A. Wixforth, Phys. Rev. Lett. 82, 2171 (1999)

[19] P. Hess, Phys. Today 55, 42 (2002)

[20] A.M. Lomonosov, P. Hess, A.P. Mayer, Phys. Rev. Lett 88, $076104(2002)$

[21] A.P. Mayer, Ultrasonics 48, 478 (2008)

[22] S. Hermelin, S. Takada, M. Yamamoto, S. Tarucha, A.D. Wieck, L. Saminadayar, C. Bäuerle, T. Meunier, Nature 477, 435 (2011)

[23] R.P.G. McNeil, M. Kataoka, C.J.B. Ford, C.H.W. Barnes, D. Anderson, G.A.C. Jones, I. Farrer, D.A. Ritchie, Nature 477, 439 (2011)

[24] M.G. Velarde, J. Comput. Appl. Maths. 233, 1432 (2010)

[25] M.G. Velarde, A.P. Chetverikov, W. Ebeling, E.G. Wilson, K.J. Donovan, Eur. Phys. Lett. EPL 106, 27001 (2014)

[26] M.G. Velarde, Eur. Phys. J. ST 225, 921 (2016)

[27] J.-P. Launay, M. Verdaguer, Electrons in molecules from basic principles to molecular electronics (Oxford Univ. Press, 2013)

[28] A.P. Chetverikov, W. Ebeling, M.G. Velarde, Eur. Phys. J. B 80, 137 (2011); 89, 196 (2016)

[29] A.V. Savin, Y.S. Kivshar, B. Hu, Phys. Rev. B 82,
$195422(2010)$

[30] M. Toda, Theory of Nonlinear Lattices, 2nd edn. (Springer-Verlag, New York, 1989)

[31] A.M. Iskandarov, N.N. Medvedev, P.V. Zakharov, S.V. Dmitriev, Comput. Mat. Sci. 47, 429 (2009)

[32] S.V. Dmitriev, E.A. Korznikova, Yu.A. Baimova, M.G. Velarde, Physics-Uspekhi 59, 446 (2016)

[33] M.G. Velarde, A.P. Chetverikov, W. Ebeling, S.V. Dmitriev, V.D.Lakhno, Eur. Phys. J. B 89, 101 (2016)

[34] A.A. Minzoni, N.F. Smyth, Wave Motion 24, 291 (1996)

[35] A.P. Chetverikov, W. Ebeling, M.G. Velarde, Wave Motion 48, 753 (2011)

[36] A.S. Davydov, Solitons in Molecular Systems, 2nd edn. (Reidel, Dordrecht, 1991)

[37] A.P. Chetverikov, W. Ebeling, M.G. Velarde, Eur. Phys. J. B 85, 291 (2012)

[38] D. Hennig, A. Neissner, M.G. Velarde, W. Ebeling, Phys. Rev. E 73, 024306 (2006)

[39] D. Hennig, A.P. Chetverikov, M.G. Velarde, W. Ebeling, Phys. Rev. E 76, 046602 (2007)

[40] L. Brizhik, A.P. Chetverikov, W. Ebeling, G. Röpke, M.G. Velarde, Phys. Rev. B 85, 245105 (2012)

[41] O.G.Cantu-Ross, L. Cruzeiro, M.G. Velarde, E. Ebeling, Eur. Phys. J. B 80, 545 (2011) 